\title{
Energy Conservative Limit Cycle Oscillations
}

\author{
Stefano Stramigioli ,Michel van Dijk \\ University of Twente, P.O.Box 217, Enschede, The Netherlands, \\ S.Stramigioli@utwente.nl
}

\begin{abstract}
This paper shows how globally attractive limit cycle oscillations can be induced in a system with a nonlinear feedback element. Based on the same principle as the Van der Pol oscillator, the feedback behaves as a negative damping for low velocities but as an ordinary damper for high velocities. This nonlinear damper can be physically implemented with a continuous variable transmission and a spring, storing energy in the spring when the damping is positive and reusing it when the damping is negative. The resulting mechanism has a natural limit cycle oscillation that is energy conservative and can be used for the development of robust, dynamic walking robots.
\end{abstract}

\section{INTRODUCTION}

The results described in this paper are motivated by the search for robust, energy efficient walking robots. Over the last decade, researchers around the world in both industry and universities have been working on walking robots and succeeded in building numerous working examples Duindam [2006], Wisse [2004]. While industry mainly focuses on so-called static walkers such as Honda's Asimo and Sony's QRIO, some universities based their research on dynamic walking of which examples can be found in Dertien [2005], Collins and Ruina [2005], Wisse and van Frankenhuyzen [2003]. The benefit of dynamic walking is that it exploits the natural dynamics of the mechanics of the walker, which results in highly energy efficient and natural looking locomotion.

Research on dynamic walking was initiated by McGeer McGeer [1990] in the early nineties. Originally inspired by toys, he developed several passive walking mechanisms that could walk down a shallow slope only powered by gravity. From his results the view emerged that dynamic walkers could be created based on the same principle, with the addition of actuators to provide energy instead of using gravity.

The stable gait of a dynamic walker can be interpreted as a stable limit cycle of the system Wisse [2004]. Once the walker has converged to the stable gait it keeps repeating the same pattern over and over again. Unfortunately the dynamic walkers that have been built so far suffer from a lack of robustness. The stability of the gate is easily destroyed by even relatively small disturbances, usually resulting in the robot falling down. Apparently the limit cycle of the system, although being stable, has only a narrow area of attraction. Current research is focused on improving this shortcoming and is expected to yield more robust behavior.

The dynamics of a walking robot are generally nonlinear and on top of that the regular impacts with the ground causes a switching behavior that makes it hard to understand the dynamics of these systems in an analytical way. This explains why the current generation of dynamic walkers is more often a result of trial and error and parameter optimization rather than a thorough analysis of the dynamic behavior that is responsible for the stable limit cycle oscillation. Although also this paper does not give a full analysis of the nonlinear dynamics, a new approach is taken in the design of dynamic walkers that focuses on generation of stable limit cycles. Inspired by nonlinear oscillators famous for their globally attractive limit cycles such as the Van der Pol oscillator, a new way is proposed for inducing limit cycle oscillation in mechanisms based on energy feedback. The result described in this paper is a mechanism that has a natural limit cycle oscillation, is energy efficient and on top of that fairly easy to implement. It is expected that this concept will enable us to build robust, dynamic walkers that excel in a combination of simplicity and performance.

The remainder of this paper is organized as follows. Section 2 introduces some necessary background information on nonlinear oscillators that exhibit stable limit cycle oscillations. In section 3 an implementation is proposed that is energy conservative, based on the theory of portHamiltonian systems. In section 4 an example is given of how the proposed implementation can be used in a physical system. Finally in section 5 this paper is concluded and future research on this subject is discussed.

\section{LIMIT CYCLES AND NONLINEAR OSCILLATORS}

A limit cycle is a periodic solution of a differential equation with the additional property that it is isolated. In the phase space of the system a periodic solution is a trajectory that is a closed orbit. Isolated means that any neighboring trajectory of the limit cycle is not closed, they spiral either towards or away from the limit cycle. Mathematically it could also be said that there exists an open neighborhood that contains only one periodic solution. If all neighboring trajectories spiral towards the limit cycle it is stable or attractive, otherwise it is unstable or half-stable in some exceptional cases. For the design of robust walking robots it is interesting to look at stable limit cycles, with a basin 
of attraction that is as large as possible. The possibility of a limit cycle solution is restricted to nonlinear systems. In a linear system, if $x(t)$ is a solution then because of linearity also $c \cdot x(t)$ is a solution for any constant $c$. In the phase space this can be seen as an infinite number of closed trajectories encircling the single equilibrium point in the origin, however non of these trajectories is isolated.

\subsection{Lienard systems}

There exist nonlinear systems which are known to have a globally attractive limit cycle. An example is the famous Van der Pol oscillator that is described by:

$$
\ddot{x}+\mu\left(x^{2}-1\right) \dot{x}+K x=0 .
$$

It was discovered by the Dutch scientist Balthasar van der Pol during the early development of radio technology in which vacuum tubes were used. The equation is similar to the damped harmonic oscillator but with a nonlinear damping term $\mu\left(x^{2}-1\right) \dot{x}$. For positive $\mu$ the damping term is negative for $|x|<1$ and positive for $|x|>1$. This results in small amplitude oscillations being pumped up, while large amplitude oscillations are damped down. Intuitively it is understandable that this must lead to a stable oscillation of intermediate amplitude. An oscillator closely related to the Van der Pol oscillator is the somewhat less famous Rayleigh oscillators that is based on the same principle. In this oscillator the damping term only depends on the derivative $\dot{x}$, which is also what is used in the examples further on in this paper. The equation describing the Rayleigh oscillator is:

$$
\ddot{x}+\mu\left(\dot{x}^{2}-1\right) \dot{x}+K x=0 .
$$

The relation between the two oscillators described above can be found by first differentiating (2) with respect to time and then replacing $\dot{x}$ with $y$.

The Van der Pol equation is a specific case of a Lienard system as described by the equation below:

$$
\ddot{x}+f(x) \dot{x}+g(x)=0 .
$$

Here $f(x)$ and $g(x)$ may be nonlinear functions. Lienards theorem Strogatz [1994] states that (3) has a unique, stable limit cycle surrounding the origin of the phase space if the following conditions are satisfied:

(1) $f(x)$ and $g(x)$ are continuously differentiable for all $x$

(2) $g(-x)=-g(x)$ for all $x(g(x)$ is odd)

(3) $g(x)>0$ for $x>0$

(4) $f(-x)=f(x)$ for all $x(f(x)$ is even $)$

(5) The odd function $F(x)=\int_{0}^{x} f(u) d u$ has exactly one positive zero at $x=a$, is negative for $0<x<a$, is positive and nondecreasing for $x>a$, and $F(x) \rightarrow \infty$ as $x \rightarrow \infty$

The conditions on $g(x)$ ensure that its behavior is like that of a restoring force like a spring and the conditions on $f(x)$ ensure a damping behavior that is amplifies small amplitude oscillations, but damp down large amplitude oscillations. More information on nonlinear oscillators and nonlinear dynamics in general can be found in books as for example Strogatz [1994], Wiggins [1990] or Khalil [1996].

\subsection{Passivity based oscillators}

Another approach to the analysis of limit cycle oscillations is taken in Stan [2005]. Here the authors use dissipativity

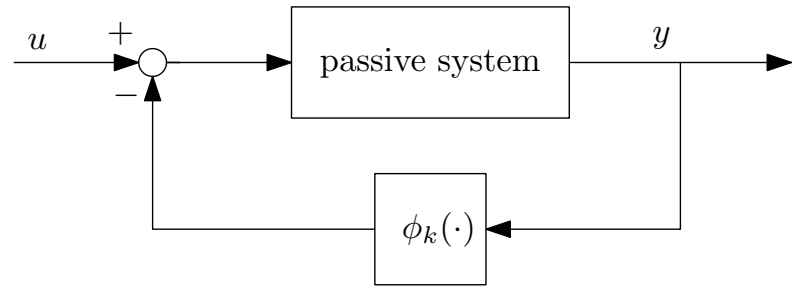

Fig. 1. Passive system with nonlinear feedback

theory to characterize oscillators as open systems. This makes it possible to interconnect a network of oscillators and analyze their common behavior. For the purposes of this paper focus is on isolated oscillators only, but the passivity approach is useful because it allows looking at system connections from an energy based point of view. A system is passive with respect to its input $u(t)$ and output $y(t)$ if there exists as storage function $S(x(t)), S(0)=0$ such that:

$$
S(x(t)) \geq 0 \quad \text { and } \quad \dot{S}(x(t)) \leq u(t) \cdot y(t) .
$$

Starting point for the analysis in Stan [2005] is the above described Van der Pol oscillator and the Fitzhugh-Nagumo oscillator, which is a simplified model of spike generation in neurons. The author generalizes these two types of oscillators to a form as shown in Fig. 1. The forward path consists of a passive system and the negative feedback is formed by a nonlinearity $\phi_{k}(y)$ that is the sum of a passive part $\phi(y)$ and an anti-passive or active part $-k y$.

$$
\phi_{k}(y)=\phi(y)-k y
$$

Here $\phi(y)$ is a smooth, static nonlinearity in the sector $(0, \infty)$ (thus passive), and moreover $\phi(y)$ is a stiffening nonlinearity, i.e. $\lim _{|y| \rightarrow \infty} \frac{\phi(y)}{y}=\infty$. Now take $G_{k}(s)$ the system formed by the linearized passive system with negative feedback $-k y$. Increasing $k$ will eventually lead to instability of $G_{k}(s)$ as poles cross the imaginary axis and move onto the right half s-plane. Define $k^{*}$ the smallest $k>0$ for which $G_{k}(s)$ has a pole on the imaginary axis. Under the assumption of absolute stability of the system shown in Fig. 1 for $k=k^{*}$ two scenarios are possible:

Scenario 1, Van der Pol type For $k=k^{*}$ a pair of complex conjugate poles cross the imaginary axis at nonzero speed causing a supercritical Hopf bifurcation. In this bifurcation the stable origin becomes unstable and a stable limit cycle emerges from the origin.

Scenario 2, Fitzhugh-Nagumo type For $k=k^{*}$ a single pole crosses the imaginary axis causing a pitchfork bifurcation that results in a bistable system. Extending the negative feedback with a slow adaptation mechanism $\frac{1}{\tau s+1}$ transforms the bistable system into a system with a globally stable limit cycle.

In the isolated case where the system is not connected $(u=0)$, the system will exhibit a self-sustained stable limit cycle oscillation for $k \gtrsim k^{*}$. The existence of this limit cycle is not guaranteed for all $k>k^{*}$ since further bifurcations may occur that alter the system behavior.

\subsection{Port Hamiltonian Systems}

Key point for the limit cycle oscillations is the nonlinear element that is locally generative, but globally dissipative. 
The interaction of the passive part of the system with the nonlinearity can be described by an exchange of energy through a power port connecting the two parts. The amount of power $P(t)$ equals the product $u(t) \cdot y(t)$ and the total exchange of energy is the time integral of the power $E(t)=\int_{0}^{t} P(u) d u$. A powerful framework for modeling dynamical systems that are described by an energy function and connections through powerports is that of port-Hamiltonian systems given by the following set of differential equations:

$$
\begin{aligned}
& \dot{x}=(J(x)-R(x)) \frac{\partial H(x)}{\partial x}+g(x) u \\
& y=g^{T}(x) \frac{\partial H(x)}{\partial x}+(K(x)-S(x)) u
\end{aligned}
$$

In these equations $x$ represents the state, $H(x)$ is the energy function or Hamiltonian, $J(x)$ and $K(x)$ are skewsymmetric matrices that model powercontinuous elements, $R(x)$ and $S(x)$ are positive semi-definite matrices that model dissipative elements and $(u, y)$ is the port through which the system can interact with the outer world. These systems have the property that $\dot{H}(x) \leq u^{T} y$, so these systems are passive with storage function $H(x)$.

\section{ENERGY CONSERVATIVE IMPLEMENTATION}

Nonlinear oscillators as the Van der Pol oscillator are generally considered nonconservative since energy dissipation takes place in the nonlinear element. When the nonlinearity behaves generatively, energy has to be supplied from an external source. However it is easy to see that for every periodic solution the change in energy of the system must be zero because energy can be expressed as a function of the state.

$$
\left.\begin{array}{l}
\Delta E=E(x(t+T))-E(x(t)) \\
x(t+T)=x(t)
\end{array}\right\} \Rightarrow \Delta E=0
$$

Therefore, instead of using a nonlinearity that dissipates energy it would be useful to have an element that buffers energy so that it can be reused later. In this section it is described how to model an element that has the same characteristics as the nonlinearity, but buffers energy instead of dissipating it. The usage of this element results in oscillators that do not dissipate any energy once converged to the stable limit cycle.

Although not commonly known, bondgraphs as introduced by Paynter Paynter [1961] can be very useful in the analysis of systems that are connected with powerports. The following analysis is based on bondgraph terminology, but is presented in a general form so that no bondgraph knowledge is required to understand the ideas presented.

\subsection{Power continuous transmissions}

To be able to shape the characteristic of the buffer a power continuous transmission (PCT) is used with transmission ratio $n$ as described by the constitutive relations below and of which a graphical representation is shown in Fig. 2.

$$
\begin{aligned}
& \text { out }_{1}=n \cdot i n_{2} \\
& \text { out }_{2}=n \cdot i n_{1}
\end{aligned}
$$

The transmission is power continuous in the sense that the power that flows into the system at one port, flows out at

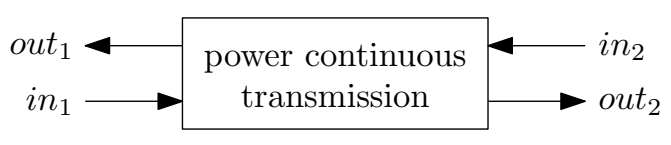

Fig. 2. Power continuous transmission

the other port in the same amount. No energy is stored or dissipated by the transmission. This property is easily deduced with the constitutive relations of (8):

$$
\begin{aligned}
P_{1} & =i n_{1} \cdot \text { out }_{1} \\
& =\left(\frac{1}{n} \cdot \text { out }_{2}\right) \cdot\left(n \cdot i n_{2}\right) \\
& =\text { out }_{2} \cdot i n_{2} \\
& =P_{2} .
\end{aligned}
$$

It is good to realize that this power continuous property still holds if the transmission factor $n$ is not constant. Such a transmission is referred to as being modulated by a factor $n(\cdot)$. In bondgraphs, this element is known as a (modulated) transformer. Physical examples of power continuous transmissions with a constant transmission ratio are an ideal electric transformer or a set of frictionless gears.

It can be shown that the modulation factor $n(\cdot)$ can be chosen such that the power flow is always in the same direction, that is choosing $n(\cdot)$ such that the PCT becomes a one way device. The positive direction of power flow is defined as the direction from $\left(i n_{1}\right.$, out $\left._{1}\right)$ to $\left(i n_{2}\right.$, out $\left._{2}\right)$. In perspective of Fig. 2, power flows from left to right if $i n_{1} \cdot$ out $_{1}>0$ and consequently from right to left if $i n_{1} \cdot$ out $_{1}<0$. Suppose $n=i n_{1} \cdot i n_{2}$ is taken as modulation factor, resulting in:

$$
\begin{aligned}
P_{1}=i n_{1} \cdot \text { out }_{1} & =i n_{1} \cdot n \cdot i n_{2} \\
& =i n_{1}^{2} \cdot i n_{2}^{2} \\
& \geq 0 .
\end{aligned}
$$

As can be seen the flow of power is always in the positive direction with this modulation factor. Similarly, the flow is always in the negative direction if $n=-i n_{1} \cdot i n_{2}$ is taken. The structure of the PCT with this modulation is depicted in Fig. 3.

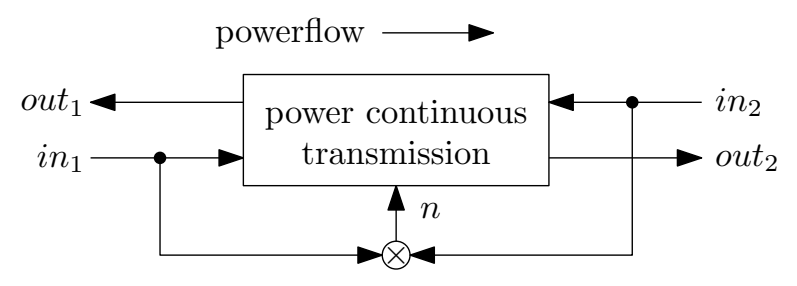

Fig. 3. Modulated PCT, with powerflow from port 1 to port 2

\subsection{Storage element}

Besides the power continuous transmission to guide the flow of power also a storage element is needed to store and supply the energy associated with the power flows, which in bondgraph terms is implemented by I- or Ctype buffers. Such a storage element can be modeled by a simple integrator as shown in Fig. 4. In the storage element the input is integrated to obtain a state $x$ and the output equals the partial derivative of the stored energy 


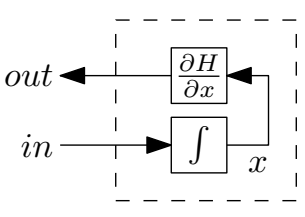

Fig. 4. Storage element

with respect to the state. In the linear case this simplifies to $\frac{x}{C}$, where the constant $C$ represents the capacity of the storage element. This describes for example a spring with the velocity as input, length as state, capacity $\frac{1}{K}$ and output the force, or a mass with the force as input, impulse as state, mass as capacity and velocity as output. The amount of energy stored in the element is found by calculating the integral over the product of the input and output.

$$
\int \text { in } \cdot \text { out } d t=\int \frac{\partial H}{\partial x} \dot{x} d t=H(x)
$$

In the linear case this equals:

$$
H(x)=\int \frac{x}{C} \dot{x} d t=\frac{1}{2 C} x^{2} .
$$

\subsection{Replacing the nonlinear element}

It is now possible to show how the combination of a modulated power continuous transmission and a storage element can be used as a substitution for the nonlinear element. Starting from the nonlinearity $\phi(\cdot)$ where the output is a nonlinear function of the input, that is:

$$
\text { out }=\phi(\text { in })
$$

and using a structure as shown in Fig. 5, the following relation can be deduced:

$$
\left.\begin{array}{l}
\text { out }=n \cdot b_{\text {out }}=n \cdot \frac{\partial H}{\partial x} \\
\text { out }=\phi(\text { in })
\end{array}\right\} \quad n=\frac{\phi(\text { in })}{\frac{\partial H}{\partial x}}
$$

That is the structure of Fig. 5 where modulation factor $n$ is according to (14) will have the same input-output characteristic as nonlinearity $\phi(\cdot)$. When the power inflow is positive and $\phi(\cdot)$ behaves as a dissipative element, now energy is stored in the buffer instead of being dissipated. When $\phi(\cdot)$ would have a generative characteristic, the buffer supplies the previously stored energy. It can do so as long as there is energy stored in to buffer, that is as long as $\frac{\partial H}{\partial x}>0$. At the same time it is clear that $\phi(\cdot)$ can be freely chosen, so it is possible to replace any nonlinearity with this system. In section 4 an example and possible physical implementation of this system is given.

\subsection{Conservative Van der Pol oscillator}

Using the buffer as sketched above to replace the nonlinear damping term in the Van der Pol equation, it can be

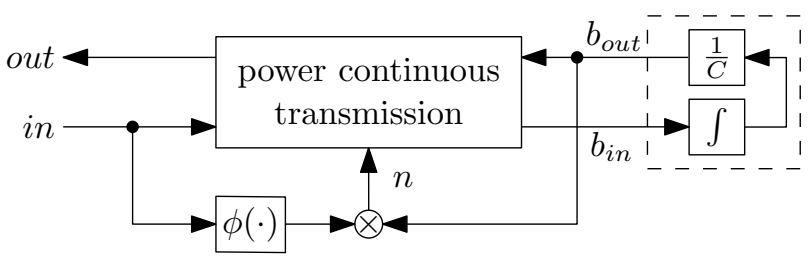

Fig. 5. Modulated PCT with storage element

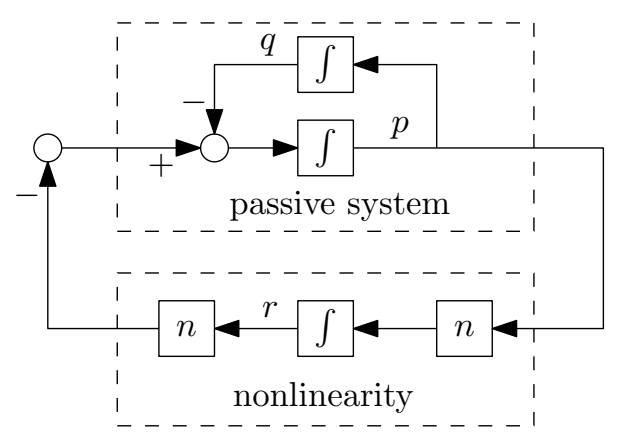

Fig. 6. Van der Pol oscillator in conservative form

written in a form that is conservative. First the Van der Pol oscillator is rewritten to equal the form of Fig. 1.

$$
\ddot{x}+\phi(x, \dot{x})+x, \quad \phi(x, \dot{x})=\mu\left(x^{2}-1\right) \dot{x}
$$

A block diagram of the system is shown in Fig. 6 where the following variables are used: $q=x, p=\dot{x}, r$ the state of the storage element with capacity $C=1$ and $n=\phi(\cdot) / r$. The equations describing the system in matrix form are:

$$
\left[\begin{array}{c}
\dot{q} \\
\dot{p} \\
\dot{r}
\end{array}\right]=\left[\begin{array}{ccc}
0 & 1 & 0 \\
-1 & 0 & -n \\
0 & n & 0
\end{array}\right]\left[\begin{array}{l}
q \\
p \\
r
\end{array}\right] \text {. }
$$

Which is a port-Hamiltonian system with state vector $x$ and skew-symmetric matrix $J$ denoted by:

$$
x=\left[\begin{array}{lll}
q & p & r
\end{array}\right]^{T} \quad J=\left[\begin{array}{ccc}
0 & 1 & 0 \\
-1 & 0 & -n \\
0 & n & 0
\end{array}\right] .
$$

The Hamiltonian of the system is:

$$
H(x)=\frac{1}{2} x^{T} \cdot x=\frac{1}{2} q^{2}+\frac{1}{2} p^{2}+\frac{1}{2} r^{2} .
$$

This is a conservative system as can be seen by calculating the time derivative of $H(x)$ :

$$
\begin{aligned}
\dot{H}(x) & =\frac{\partial H}{\partial x} \dot{x} \\
& =x^{T} J x \\
& =0
\end{aligned}
$$

where equality to zero follows from the skew-symmetric property of $J$.

It can be verified that the behavior of the system in conservative form is the same as that of the normal Van der Pol oscillator by a numerical simulation. In Fig. 7 a solution of the system for $\mu=1$ is shown with initial conditions $x=[2,2,10]$. The figure shows convergence to a limit cycle in the $(q, p, r)$-space, and the projection of the trajectory on the $(q, p)$-surface shows the limit cycle associated with the normal Van der Pol oscillator, thereby verifying that the behavior of the conservative system is indeed the same.

\subsection{Extension to systems with dissipation}

In the previous example the passive part consists of conservative elements only. If the passive part contains also dissipative elements then the total energy flow into the nonlinearity will be smaller than the amount of energy it has to supply. As a result the stored energy in the buffer will decrease as time evolves until the buffer becomes empty. To overcome such problems energy has to be injected into the system that compensates the dissipation 


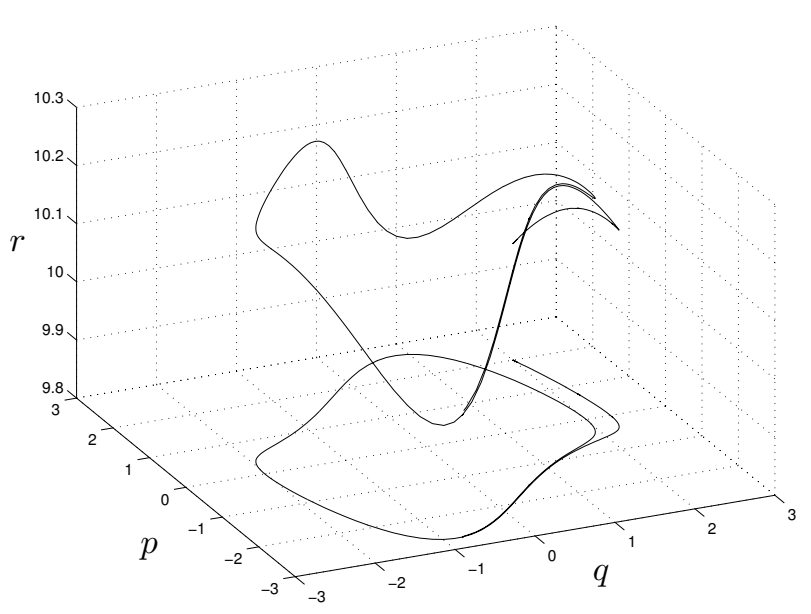

Fig. 7. Simulation of the conservative Van der Pol implementation

of energy in the passive part. Connecting an actuator directly to the system however will generally influence the dynamics of the system and thereby possibly disturb the stability properties of the limit cycle. With the use of the storage element it is possible to circumvent this problem in an elegant way. Instead of injecting energy in the system, it is possible to directly inject energy into the storage element. As the modulation factor of the power continuous transmission compensates for any variation of energy storage this can be done without influencing the system behavior. Energy injection can be done by extending the storage element to a two port system as shown in Fig. 8 where port 1 connects to the system

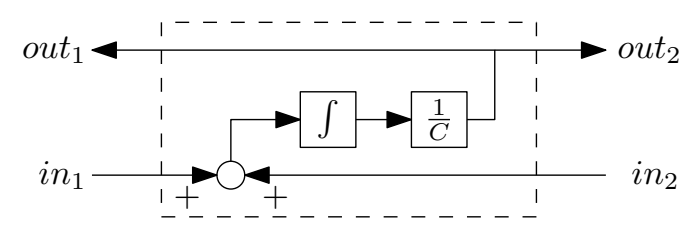

Fig. 8. Two port storage element

through the power continuous transmission as before and port 2 is used to supply the required energy. If the storage element is implemented as a torsional spring for example, one side of the spring connects to the system and the other side to a motor which winds up the spring similarly like what is done in an analogue wristwatch.

\section{APPLICATIONS AND IMPLEMENTATION}

The last part of this paper discusses an application and possible physical implementation of the system described so far. As the motivation for this paper comes from research on walking robots the application will be in that field. The leg of a walking robot can be roughly interpreted as an inverse pendulum, or double inverse pendulum in case of legs with knees and therefore it is chosen to look at how limit cycle oscillations can be induced in a pendulum.

\subsection{Pendulum}

The pendulum is a classic physical example of a nonlinear differential equation. The differential equation describing the damped pendulum of Fig. 9 with pointmass $m$, length $l$, damping $d$ and input torque $T$ is:

$$
\ddot{\theta}+\frac{d}{m \cdot l} \dot{\theta}+g \sin \theta-\frac{T}{m \cdot l}=0 .
$$

The system is passive with respect to input $T$ and output $\dot{\theta}$, so limit cycle oscillation is expected if a negative feedback of the form $-T=\dot{\theta}^{3}-k \dot{\theta}$ is used. Although not strictly a Lienard system, the system with this feedback is similar to the Rayleigh oscillator and the nonlinearity fulfills the conditions described in section 2 . It can be seen that the term $k \dot{\theta}$ will compensate the damping term $\frac{d}{m \cdot l} \dot{\theta}$ and thus limit cycle oscillation may be expected for $k>\frac{d}{m \cdot l}$. That is, the system is stable for small $k$, increasing $k$ results in a Hopf-bifurcation in which the stable equilibrium becomes unstable and a globally stable limit cycle surrounding the origin appears.

These expectations are verified by numerical simulation of which the results are shown in Fig. 10 and 11. The parameters used in the simulation are: $m=1, l=1, d=2$ and $g=9.81$. In Fig. $10 k=1$, which results in a damped, stable system as the damping of the pendulum is stronger than the active part of the nonlinear feedback. Increasing $k$ results in a limit cycle oscillation as is shown in Fig. 11 for a value $k=4$.

\subsection{Implementation}

In order to build the pendulum with nonlinear feedback in a power continuous way, a physical implementation for the modulated power continuous transmission and storage element have to be found. The (input, output) combinations of the transmission are of the form (rotational velocity, torque) so the device must transform rotation to rotation.

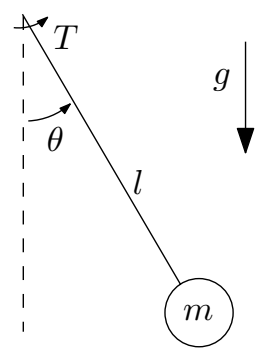

Fig. 9. Classic pendulum

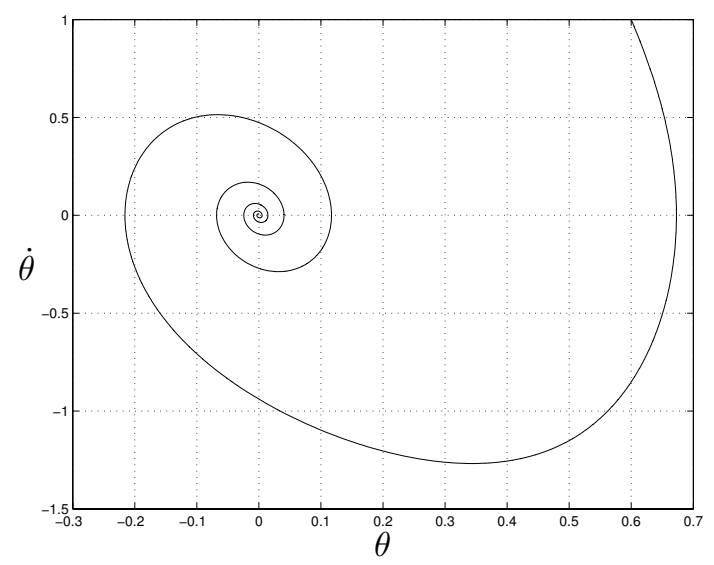

Fig. 10. Damped pendulum 


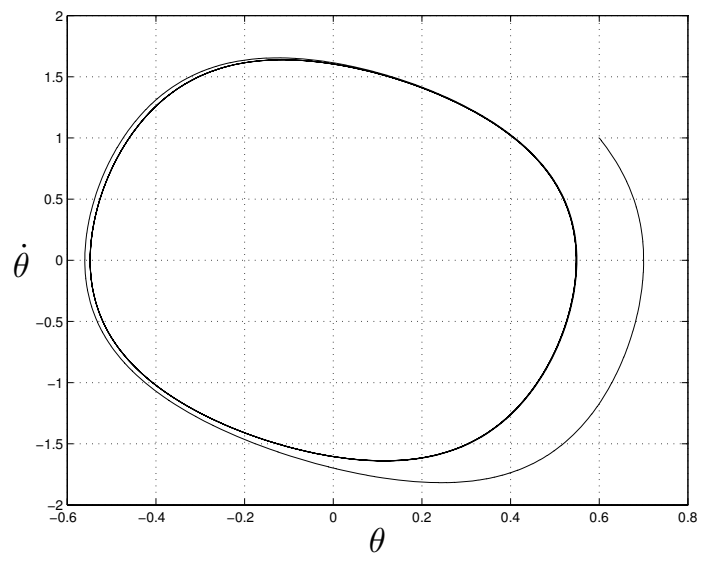

Fig. 11. Pendulum in limit cycle

A set of gears would suffice if the transmission ratio would be constant, but in this case the ratio must be variable. A physical device with a variable transmission ratio is called a continuous variable transmission (CVT). There are several possible implementations to create a physical CVT. In the automobile industry it is most common to use two adjustable pulleys connected by a steel belt. The pulleys are created in such a way that their radius can be increased by compressing the pulley. The change of the radius results in a different transmission ratio. Another possible implementation is schematically drawn in Fig. 12. This implementation consists of a pair of lined up conic cylinders that are connected by a belt. By adjusting the position of the belt on the cylinders with active control, a continuous range of transmission ratios can be selected. These are just schematic ideas. In reality the coefficient

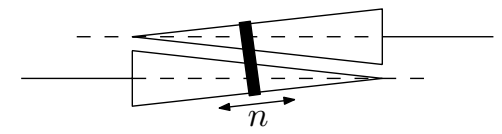

Fig. 12. CVT implementation with conic cylinders

$n$ should vary between negative and positive values and this can be achieved with a planetary gear. This is at the moment under study and conceptual designs are available. The basic idea is to create a CVT with $n \in\left[n_{\min }, n_{\max }\right]$ creating a speed relation $\omega_{1}=n \omega_{2}$. Furthermore, we can create a gearing system realizing a relation with an additional axis of the form $\alpha \omega_{1}=\beta \omega_{2}+\omega_{3}$, which together with the CVT would realize a relation between $\omega_{1}$ and $\omega_{3}$ as:

$$
\omega_{3}=m \omega_{2} \quad m:=(\alpha n-\beta) .
$$

This implies that by choosing proper fixed gear rations $\alpha$ and $\beta$, we can achieve a desired range $m \in\left[m_{\min }, m_{\max }\right]$ for negative $m_{\min }$ and positive $m_{\max }$ :

$$
\left(\begin{array}{l}
\alpha \\
\beta
\end{array}\right)=\left(\begin{array}{ll}
n_{\min }-1 \\
n_{\max }-1
\end{array}\right)^{-1}\left(\begin{array}{l}
m_{\min } \\
m_{\max }
\end{array}\right)
$$

\section{CONCLUSIONS AND FUTURE WORK}

It was shown how stable limit cycle oscillations can be induced in a passive system with the use of a nonlinear feedback. Similarly to the Van der Pol oscillator, this feedback pumps up small amplitude oscillation, but damps down large amplitude oscillation thus resulting in a stable oscillation of intermediate amplitude. The passive system and the nonlinear feedback continuously exchange energy. Whereas in the Van der Pol oscillator energy that flows into the nonlinear feedback is dissipated, it was shown how to convert this into a conservative system. With the combination of a modulated power continuous transmission and a storage element any nonlinear characteristic can be implemented by choosing the appropriate modulation factor. Energy that otherwise would be dissipated can now be reused and fed back to the system. It was also shown how the concept can be extended with a two port storage element to compensate for energy losses in the passive part of the system.

Systems that exhibit stable limit cycle oscillation are interesting for the development of robust, dynamic walking robots. Future research will focus on how the concept described in the paper can be implemented in a walking robot. It would be interesting to analyze what the exact influence of the nonlinear feedback is on the shape of the resulting limit cycle. Other points of interest are how the system can be generalized to higher dimensions and how the oscillation can be synchronized with impacts of the feet with the ground.

\section{ACKNOWLEDGEMENTS}

The authors would like to acknoledge the input and contribution for the study on the CVT of Jos Ansink.

\section{REFERENCES}

Steve Collins and Andy Ruina. A bipedal walking robot with efficient and human-like gait. IEEE International Conference on Robotics and Automation, pages 19831988, 2005.

Edwin Dertien. Realisation of an energy-efficient walking robot. Master's thesis, University of Twente, june 2005.

Vincent Duindam. Port-Based Modeling and Control for Efficient Bipedel Walking Robots. PhD thesis, University of Twente, Maart 2006.

Hassan K. Khalil. Nonlinear Systems. Prentice-Hall, 1996.

Tad McGeer. Passive dynamic walking. International Journal of Robotics Research, 9(2):62-82, 1990.

Henry A. Paynter. Analysis and Design of Engineering Systems. M.I.T. Press, 1961.

Guy-Bart Stan. Global analysis and synthesis of oscillations: a dissipativity approach. $\mathrm{PhD}$ thesis, Universite de Liege, March 2005.

Steven H. Strogatz. Nonlinear Dynamics and Chaos. Addison-Wesley, 1994.

Stephen Wiggins. Introduction to Applied Nonlinear Dynamical Systems and Chaos. Number 2 in Texts in Applied Mathematics. Springer-Verlag, 1990.

Martijn Wisse. Essentials of dynamic walking. PhD thesis, Delft University, September 2004.

Martijn Wisse and Jan van Frankenhuyzen. Design and construction of mike: a $2 \mathrm{~d}$ autonomous biped based on passive dynamic walking. International Conference on Adaptive Motion of Animals and Machines, 2003. 